\title{
The role and the signal pathways of Yes-associated protein 2 in hypertrophic cardiomyopathy
}

\author{
Bangrong Song, Haiming Dang and Ran Dong \\ Department of Cardiac Surgery, Beijing Anzhen Hospital, Capital Medical University, Beijing, China
}

\begin{abstract}
Hypertrophic cardiomyopathy (HCM) is a heterogeneous myocardial disease characterized by myocardial hypertrophy, myocardial mechanical and electrical activity obstacles. This study aimed to explore the relationship between YAP2 (Yes-associated protein 2) and HCM and clarify a signaling path about the pathogenesis of HCM. Our study confirms that YAP2 can promote myocardial cell hypertrophy at the molecular level (myocardial lineage cell H9C2), organ level (clinical specimens of human HCM), and an animal model (a mouse model of HCM with cardiac-specific transgenic and knockout YAP2). The detailed molecular mechanisms linking YAP2 to cardiomyocyte hypertrophy and HCM were investigated. This study proved that YAP2, as the final reaction factor in Hippo pathways, influences Akt1 activity to affect the downstream genes, which participate in the formation of HCM by promoting myocardial cell proliferation and cardiac hypertrophy.
\end{abstract}

Key words: YAP2 - Hypertrophic cardiomyopathy - Myocardial cells specific transgenic rats - AKT1

\section{Introduction}

Cardiovascular disease has become the leading cause of death in humans, accounting for one-third of worldwide deaths (Wong et al. 2019). A prospective study on the cause of death of ordinary adults over 40 in China shows that heart disease has become the leading cause of death for residents ( $\mathrm{He}$ et al. 2005). As a severe cardiovascular disease, hypertrophic cardiomyopathy (HCM) is getting attention, and its aetiology and related molecular mechanisms have also become the focus of current research.

$\mathrm{HCM}$ is heterogeneous cardiomyopathy characterized by myocardial hypertrophy, which can arise from various causes (such as genetic et al.). This disease is accompanied by myocardial mechanical and/or cardiac electrical activity disorders and often manifests as inappropriate ventricular hypertrophy, which can lead to cardiac insufficiency or cardiovascular death in patients. HCM can be confined to

Correspondence to: Ran Dong, Department of Cardiac Surgery, Beijing Anzhen Hospital, Capital Medical University, No. 2 Anzhen Street, Chaoyang District, Beijing 100029, China

E-mail: dongran6618@126.com the heart itself, or it can be part of systemic disease. Also, it can lead to impaired blood supply to the myocardium and peripheral blood vessels in patients, and death can occur in severe cases. In the literature, the mechanism of HCM formation has been reported with various hypotheses of signal transduction pathways. However, many of the molecular mechanisms related to HCM are not fully understood (Gangadharan et al. 2017).

Current research shows that the formation mechanism of HCM is diversified, involving mutations of multiple genes. Molecular genetic studies have identified mutations in genes encoding structural proteins of muscle groups as the underlying cause of HCM, and a mutation in one of the regulatory genes can lead to the development of the disease. There are increasing studies dealing with the signal pathways causing cell volume increase and apoptosis. The researched signal pathways include MAPK, Hippo, PI3K/Akt, and other signal pathways (Gangadharan et al. 2017). Yes-associated protein (YAP) is located at 11q22 in the genome (Overholtzer et al. 2006). The function initially discovered by YAP is its use as a transcription factor cofactor. The most crucial transcription factor associated with it is the TEAD family. As the final response factor of the signal transduction pathway MST1-

(c) The Authors 2021. This is an open access article under the terms of the Creative Commons Attribution-NonCommercial 4.0 International License (https://creativecommons.org/licenses/by-nc/4.0/), which permits non-commercial use, distribution, and reproduction in any medium, provided the original work is properly cited. 
LATS-TEAD/YAP, YAP determines the size of organ formation (Azad et al. 2019). There are a large number of reports on the Hippo pathway. These studies have found that the Hippo pathway plays an essential role in human liver cancer, breast cancer, and cervical cancer (Pan 2007; Steinhardt et al. 2008; Zhao et al. 2008, 2010; Hall et al. 2010; Liu et al. 2010; Wang et al. 2010; Avruch et al. 2011; Zhang et al. 2011). Currently, no studies on the Hippo pathway and its downstream genes in human heart tissue have been reported.

The Hippo-Lats-Yorkie pathway is a conserved pathway from Drosophila to mammals, and the study of the function and mechanism of this pathway has always been a hot spot. As a member of the MST pathway, Lats can regulate the size of organs (Jiang et al. 2009). In heart tissue, the main Lats is Lats2. In DN-Lats2 transgenic mice with functional inactivation but inhibiting endogenous Lats function, the volume of myocardial cells in a single mouse can be increased, leading to ventricular hypertrophy in mice and some pathological changes in function (Matsui et al. 2008; Zhang et al. 2008). The current literature reports have not involved the study of YAP2 and the formation of heart size, and the molecular signaling mechanism related to pathogenicity.

In this study, we constructed a mouse model of HCM with cardiac-specific transgenic and knockout YAP2, and studied and analyzed clinical specimens of human HCM. In this way, the detailed molecular mechanisms linking YAP2 to cardiomyocyte hypertrophy and HCM were investigated to clarify the role of YAP2 in myocardial hypertrophy, reveal the molecular signal transduction mechanism of YAP2 to promote cardiomyocyte hypertrophy and elucidate the molecular mechanism of HCM. It can provide a theoretical basis for diagnosing and treating clinical HCM and finding the target of drug treatment from the molecular level.

\section{Material and Methods}

\section{Research objectives}

In the study, 15 cases of interventricular septal tissue and 3 healthy left ventricular tissue cases from March 2009 to March 2011 were collected. The tissue of patients with hypertrophic myocardium is derived from the interventricular septum in the surgical treatment of HCM, and the normal control tissues are derived from the donor heart tissue during heart transplantation. All patients underwent 12-lead ECG, body surface echocardiography, esophageal echocardiography, chest radiography, and other examinations before the operation and signed informed consent. This study was approved by the Ethics Committee of Beijing Anzhen Hospital, Capital Medical University.
Preservation and processing of myocardial tissue samples: after obtain the myocardial tissues of HCM, it was washed with PBS solution, divided into two parts, wrapped in foil, and placed in a cryotube $-160^{\circ} \mathrm{C}$ and stored under liquid nitrogen. A portion of the tissue was used to extract total RNA and perform PCR experiments, and another portion was used to obtain proteins for Western blot.

\section{Preparation and detection of $m R N A$}

The TRIZO was purchased from Invitrogen Corporation, and DEPC (diethylpyrocarbonate) was purchased from Biodee Diagnostic Corporation. The RNase-free small centrifuge tubes and Tips were purchased from BYK Chemie.

\section{Total RNA extraction}

(1) Specimen homogenization. The tissues were ground on ice with a tissue homogenizer to no more visible large pieces of tissue, and the homogenized sample was centrifuged at $4^{\circ} \mathrm{C}$ and $12000 \mathrm{rpm}$ for $15 \mathrm{~min}$. After centrifugation, the supernatant was transferred to a new small centrifuge tube and incubated at $15-30^{\circ} \mathrm{C}$ for 5 min to completely decompose the ribosome. (2) Separation. For every $1 \mathrm{ml}$ of Trizol, $0.2 \mathrm{ml}$ of chloroform was added; the sample tube was caped tightly and shaken vigorously for $15 \mathrm{~s}$, then incubated at $30^{\circ} \mathrm{C}$ for $3 \mathrm{~min}$ and centrifuged at $12,000 \mathrm{rpm}$ at $2-8^{\circ} \mathrm{C}$ for $15 \mathrm{~min}$. After centrifugation, the mixture was divided into lower, middle, and upper layers. RNA was present in the water sample layer. (3) RNA precipitation. The aqueous layer was transferred to a clean test tube and mixed with isopropyl alcohol to precipitate RNA. Isopropanol was added in proportion to $0.5 \mathrm{ml}$ of isopropanol per $1 \mathrm{ml}$ of Trizol during initial homogenization. The mixed samples were incubated at $15-30^{\circ} \mathrm{C}$ for $10 \mathrm{~min}$ and centrifuged at $2-8^{\circ} \mathrm{C}$ at $12,000 \mathrm{rpm}$ for $10 \mathrm{~min}$. After centrifugation, the RNA pellet was visible, formed a gel-like pellet attached to the wall and bottom of the tube. (4) RNA elution. The upper suspension was removed and the RNA pellet was washed with ethanol in the proportion of $0.5 \mathrm{ml} 75 \%$ ethanol per $1 \mathrm{ml}$ Trizol during the initial homogenization. The samples were mixed by vortexing and centrifuged at a high speed of not more than $7,500 \times g$ for $5 \mathrm{~min}$ at $2-8^{\circ} \mathrm{C}$, the steps were repeated. (5) RNA redissolution. The RNA pellet was simply dry and dissolved in 50-80 $\mu$ l of RNase-free sterile water. (6) Agarose gel electrophoresis was used to test the integrity of RNA samples. UV spectrophotometer was used to test the concentration and purity of RNA samples.

\section{cDNA preparation}

Reverse transcriptase (M-MLV RT) and $5 \times$ reaction buffer were purchased from Promega; Oligo dT and dNTP were 
purchased from Takara. The specific steps were: (1) The following reaction mixture was added to a small centrifuge tube in an ice bath: total template RNA $2 \mu \mathrm{g}$ and Oligo(dT) 18 $(0.5 \mu \mathrm{g} / \mu \mathrm{l}) 1 \mu \mathrm{l}$, and diluted to $15 \mu \mathrm{l}$ with RNase-free ddH2O. (2) After gently mixing and after $70 \mathrm{~min}$ at $70^{\circ} \mathrm{C}$ water bath, it was placed in the ice bath for $30 \mathrm{~s}$. (3) The test tube was put on an ice bath and the following components were added separately: $5 \mu$ l of $5 \times$ reaction buffer, $1 \mu$ of M-MLV RT (200 U/ $\mu \mathrm{l})$ and $1 \mu \mathrm{l}$ of dNTP Mix ( $10 \mathrm{mmol} / \mathrm{l})$, mixed gently and centrifuged 3-5 s, diluted to $25 \mu \mathrm{l}$ with RNasefree deionized water. (4) The reaction mixture was bathed at $37^{\circ} \mathrm{C}$ for $70 \mathrm{~min}$. (5) The reaction was completed by heating at $70^{\circ} \mathrm{C}$ for $10 \mathrm{~min}$.

\section{PCR detection}

The rTaq polymerase, $5 \times$ reaction buffer, dNTP were purchased from TaKaRa Company, and the primers were synthesized by Shanghai Shengong Biological Co., Ltd. DL2000DAN molecular weight maker was purchased from Biomed. The PCR reaction parameters were: predenaturation at $94^{\circ} \mathrm{C}$ for $5 \mathrm{~min}$, denaturation at $94^{\circ} \mathrm{C}$ for $30 \mathrm{~s}$, anneal at $55^{\circ} \mathrm{C}$ or $60^{\circ} \mathrm{C}$ for $30 \mathrm{~s}$, extension at $72^{\circ} \mathrm{C}$ for $40 \mathrm{~s}$, supplemental extension at $72^{\circ} \mathrm{C}$ for $10 \mathrm{~min}$, and $28-35$ cycles.

\section{Preparation of plasmids containing fragments of interest}

The gel recovery kit and plasmid small extraction kit were purchased from Transgene; the $\mathrm{pMD}^{\mathrm{TM}} 19-\mathrm{T}$ Vector and ligation kit were purchased from TaKaRa. The amplified fragments obtained by conventional PCR were electrophoresed in $2.0 \%$ agarose gel, and TAE electrophoresis buffer was used for recovery electrophoresis, and the voltage was limited to $15 \mathrm{~V} / \mathrm{m}$. After the electrophoresis is completed, cut the desired target band under the UV lamp and then recover the target fragment according to the instructions of the gel recovery kit. After retrieved the target fragment, correct detection by agarose gel electrophoresis, the target fragment was ligated into the T vector. A $10 \mu \mathrm{l}$ reaction system was used for the ligation reaction. In the ligation reaction, the moles of the insert should be 3-10 times the moles of the carrier (depend on the length of the insert). The resultant reaction mixture was added to the small centrifuge tube: $1 \mu \mathrm{l}$ pMD19-T vector, $1 \mu \mathrm{l} \mathrm{Fragment,} 3 \mu \mathrm{l}$ water, and $5 \mu \mathrm{l}$ $2 \times$ ligation mix. The reaction was at $16^{\circ} \mathrm{C}$ for $30 \mathrm{~min}$. Note (1) The connection reaction can generally be performed at room temperature $\left(25^{\circ} \mathrm{C}\right)$, but the reaction efficiency is slightly reduced. (2) The connection reaction can be performed generally in $5 \mathrm{~min}$, but the reaction efficiency is slightly reduced. The total amount $(10 \mu \mathrm{l})$ was added to $100 \mu \mathrm{J}$ JM109 competent cells and placed on ice for 30 min. After being heated at $42^{\circ} \mathrm{C}$ for $90 \mathrm{~s}$, it was placed on ice for another minute. Add $890 \mu \mathrm{l}$ Luria-Bertani (LB) medium and incubated at $37^{\circ} \mathrm{C}$ for $60 \mathrm{~min}$ with shaking. The bacterial solution was spread on an L-agar plate medium containing X-Gal, IPTG, Amp, and cultured overnight to form single colonies; count white and blue colonies; pick white colonies and use PCR to confirm the length of the insert in the vector. The positive clones were inoculated in 5-10 ml of Amp-resistant LB liquid medium and cultured overnight at $37^{\circ} \mathrm{C}$. The next day, the plasmid was recovered according to the small plasmid kit, PCR was positive, and the plasmid concentration was determined by ultraviolet spectrophotometer.

\section{Target gene protein level detection}

\section{Extraction of total tissue protein and determination of protein concentration}

Tissue lysate strong RIPA was purchased from Biyuntian. Spectral protease inhibitor PMSF was purchased from Jingmei Company and was stored in a $10 \mathrm{mg} / \mathrm{ml}$ stock solution prepared with isopropanol, protected from light at $-20^{\circ} \mathrm{C}$. The tissue homogenizer was purchased from the supply of consumables for biophysics. The bicinchoninic acid (BCA) protein concentration determination kit was purchased from Pulilai. The multi-wavelength microplate reader was purchased from PE Company.

\section{Western blotting detection}

The membrane transfer tank was purchased from Beijing Liuyi Instrument Factory, the HRP-labeled secondary antibody was purchased from Beijing Zhongshan Company, and the ECL luminescent substrate and PVDF membrane were purchased from Millipore Company. The primary antibody was purchased from a different company, which has been stated in the text. X-ray film and exposure clips were purchased from New Huizeo.

\section{Expression of YAP2 and identification of cardiomyocyte hypertrophy}

\section{Cellular level}

Primary cardiomyocytes: We use embryonic rats of E14.5 days, take out cardiomyocytes and co-culture with YAP2 virus or U6-YAP2 RNAi virus, and get preliminary results from preliminary experiments. YAP2 overexpression can make primary cultured myocardium. There was a significant increase in the effect of the cell, and YAP2 gene knockdown can inhibit drug-induced myocardial hyperplasia. In the rats, myocardial lineage cell $\mathrm{H} 9 \mathrm{C} 2$ : after virus treatment, some genes related to myocardial hypertrophy are identified at 
the mRNA and protein levels, and specific enzyme activities are changed.

\section{Histological level}

Normal mouse traverse aortic constriction (TAC) by ligation of the transverse thoracic aorta: Changes in protein levels of YAP2 and activity as transcription factors after stimulation under physiological conditions and changes in mRNA and protein levels and some enzyme activities related to genes related to cardiac hypertrophy change were identified. Clinical specimens: Using excised cardiac specimens from patients with myocardial hypertrophy was verified whether the activity of YAP2 has changed and whether the genes regulated by YAP2 have changed at the mRNA and protein levels. There are currently ten specimens obtained.

\section{Construction of animal models}

Using the existing cardiomyocyte-specific transgenic mouse technology to obtain YAP2 cardiomyocyte-specific transgenic mouse (provided by Professor Yang Zhongzhou, Nanjing University Model Animal Center), for aortic ligation (TAC) and isoproterenol (ISO) administration the treatment method obtained an obvious myocardial hypertrophy phenotype (there are currently 10 cases of ISO treatment). At the same time, the YAP2 heart-specific knockout rats that we are about to do will also be processed in the same way to verify. Thirty Sprague-Dawley rats were randomly divided into blank control group, sham operation group, abdominal aortic constriction myocardial hypertrophy model group (TAC), and 10 rats in each group. Establishment of the rat model of myocardial hypertrophy. 20 rats in the TAC group were anaesthetized by intraperitoneal injection of $4 \%$ chloric acid hydrate solution $(60 \mathrm{mg} / \mathrm{kg})$. The rats were fixed in the supine position. A $2-3 \mathrm{~cm}$ longitudinal incision was made under the mid-abdominal xiphoid process, and the hook was pulled after entering the abdominal cavity. After opening the abdomen of the rats, the surgical field is exposed and the abdominal aorta is located. A small segment of the abdominal aorta is isolated about $3 \mathrm{~mm}$ above the branch point where the abdominal aorta sends out the right renal artery. Then, a 24-gauge needle with a diameter of $0.8 \mathrm{~mm}$ and the abdominal aorta is inserted. The arteries were ligated together with sutures, and the needle was quickly withdrawn, causing the internal diameter of the abdominal aorta to narrow at the ligation point. Finally, the abdominal organs were returned in turn, and the abdominal wall was sutured layer by layer to complete the operation. Rats in the sham group received all procedures except for aortic constriction. After the operation, the rats in the TAC group and the sham-operated group were treated with penicillin for 3 days.

\section{Modeling of rat cardiomyocyte culture and hypertrophy}

Sprague-Dawley rats, 1-3 days old, were selected, and their hearts were removed under aseptic conditions, the atria were incised, and the ventricles were cleaned with D-hanks solution. The myocardium was cut into small pieces of $1 \mathrm{~mm}^{3}$ tissue using ophthalmic forceps, and the myocardial cells were collected after digestion with trypsin, and the number of cells was counted. Serumcontaining DMEM culture medium was routinely cultured for 2 days, cultured with serum-free for $24 \mathrm{~h}$, and then given phenylephrine (PE) intervention for $48 \mathrm{~h}$ to induce cell hypertrophy.

\section{Cardiac hypertrophy cell model detection}

The myocardial hypertrophic cells in the control group's model group were stained with $\alpha$-actinin antibodies and tested with a laser confocal microscope. 100 cells were randomly selected from each group for analysis.

\section{Statistical methods}

The data obtained from the experiment were processed with Microsoft Excel and IBM SPSS Statistics 17.0 software package. The statistical value of the data is expressed as mean \pm SEM. The statistical method adopts the T-test. When the $p$-value is less than 0.05 , the data are considered to be significantly different.

\section{Results}

\section{YAP2 can promote cardiomyocyte hypertrophy}

\section{Cell detection}

The volume of primary cardiomyocytes infected with YAP2 virus knockdown was different from that of the control group (Fig. 1A). The volume of primary cardiomyocytes infected with the YAP2 virus for 3 days and 4 days was significantly different from that of GFP (control group) transfected primary cardiomyocytes (Fig. 1B). The results of co-cultivation of primary myocardium and virus show that YAP2 can make myocardial cells larger. At the cellular level, YAP2 was shown to promote cardiomyocyte hypertrophy. The volume of cardiomyocytes treated with PE in the control group increased significantly. In contrast to YAP2 knockdown cardiomyocytes and the control group with PE treatment simultaneously, the volume of YAP2 knockdown 
A

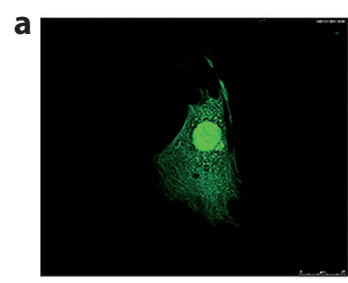

b

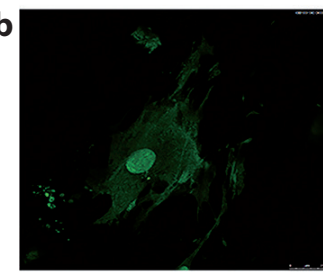

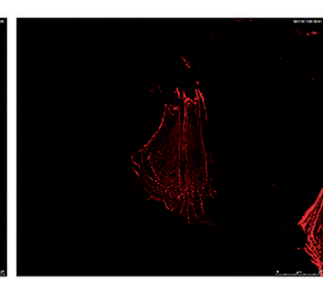

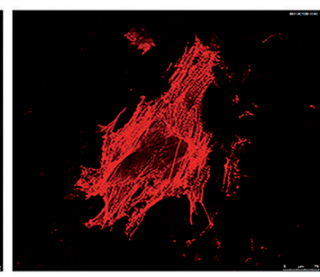

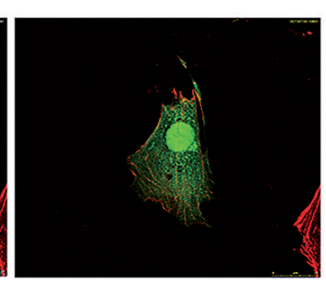

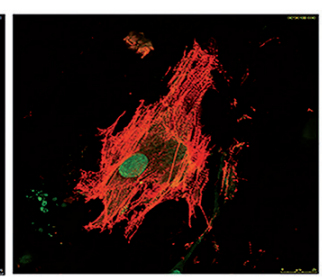

B

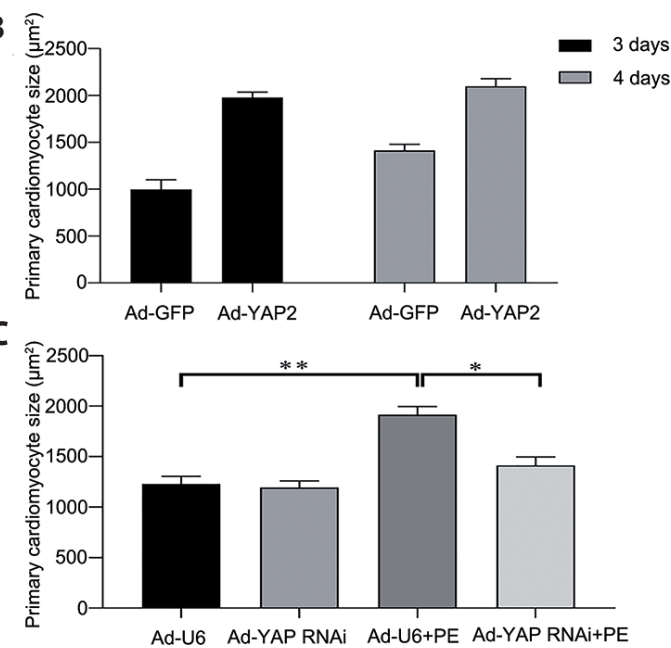

Figure 1. YAP2 promotes cardiomyocyte hypertrophy at the cellular level. A. Compared with the control group (a), the cardiomyocytes overexpressing YAP2 (b) are significantly larger. B. Statistic graph of the volume of cardiomyocytes overexpressing YAP2 and the control group cultured for 3 days and 4 days respectively. C. Cardiomyocytes knocking down YAP2 can effectively inhibit cardiomyocyte hypertrophy caused by external stimulation. Ad, adenovirus; GFP, Green fluorescent protein; YAP, Yes-associated protein; U6, promoter; RNAi, RNA interference; PE, phenylephrine. ${ }^{\star} p<0.05$; ${ }^{* *} p<0.001$.

cardiomyocytes was significantly higher than that of the control group. The cardiomyocytes knocking down YAP2 can effectively suppress the hypertrophy of cardiomyocytes caused by external stimulation (Fig. 1C).

\section{Histological analysis}

The model samples of mouse myocardial hypertrophy found that the content of YAP2 in myocardial samples of TAC rats was significantly higher than that of the control group (Fig. 2A). The samples of patients with HCM found that the content of YAP2 was higher than that of the control group (Fig. 2B).

\section{Animal model}

After seven days of ISO treatment, the hearts of normal rats and transgenic rats were collected and pathologically sliced to obtain the results shown in Fig. 3A. YAP2 was shown to promote cardiac hypertrophy at the animal model level (Fig. $3 \mathrm{~B})$. The weight of the heart of wild-type and transgenic rats was significantly different $(p<0.05)$ (Fig. 3C).

\section{Molecular mechanism of YAP2 promoting cardiomyocyte hypertrophy}

\section{Cell detection}

Cytological experiments showed that knocking down YAP2 reduces the activity of AKT, and overexpression of YAP2 can upregulate AKT activity (Fig. 4A). As shown in the figure, in passaged $\mathrm{H} 9 \mathrm{C} 2$ cardiomyocytes, YAP2 affects AKT activity. It is suggested that YAP2 can affect the downstream genes by regulating the activity of Akt1 to promote cardiomyocyte hypertrophy (Fig. 4B).
A

\section{6 weeks}

SHAM TAC

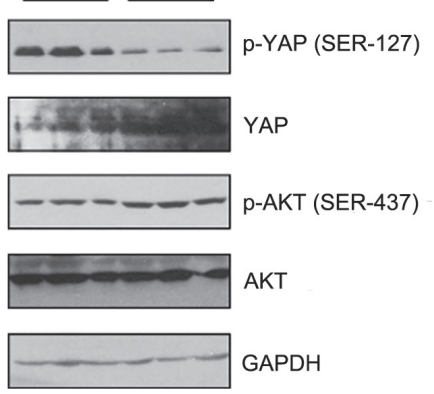

B

n1 n2 n3 h1 h2 h3

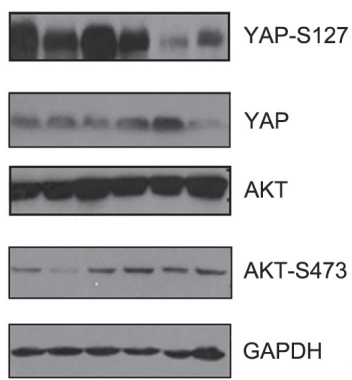

Figure 2. YAP2 promotes cardiomyocyte hypertrophy at the histological level. A. The model sample of mouse myocardial hypertrophy found that the content of YAP2 in myocardial samples of TAC was significantly higher than that of the sham group. B. Samples of patients with HCM found that the content of YAP2 was higher than that of the control group. SHAM, sham operation group; TAC, thoracic aorta ligation group; $\mathrm{n} 1-\mathrm{n} 3$, normal control group; h1-h3, cardiomyocyte hypertrophy group; LV, left ventricle; TAC, thoracic aorta ligation; six weeks, the myocardial hypertrophy time; p-AKT, Akt signaling pathway. 


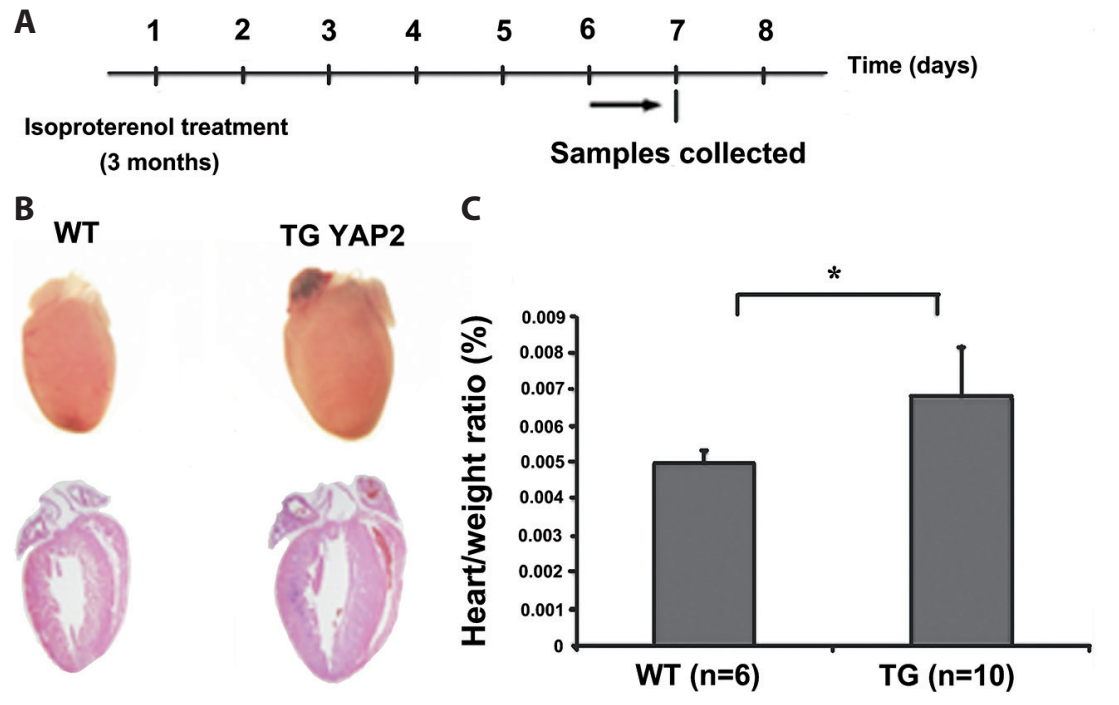

Figure 3. YAP2 promotes cardiomyocyte hypertrophy in the animal model. A. The obtained model diagram of mouse heart specimen. B. Pathological slices of normal (wild type, WT) and YAP2 heart-specific transgenic rats after ISO treatment (TG YAP2). C. The ratio of heart to body weight in normal rats (WT) and YAP2 heart-specific transgenic rats (TG). ${ }^{*} p<0.05$.
Histological analysis

A model sample of mouse cardiac hypertrophy showed that YAP2 could upregulate the activity of AKT1 (Fig. 5A). The sample of patients with HCM showed that YAP2 could upregulate the activity of AKT1 (Fig. 5B).

\section{Discussion}

The MST signaling pathway is very conserved in lower to higher animals, and the principal members include MST1/2, WW45, LATS1/2, and Mob1 (Zhou 2014; Zheng et al. 2015; Yang et al. 2018), and homologous proteins, including Hippo,

\section{A Ad-YAP2}

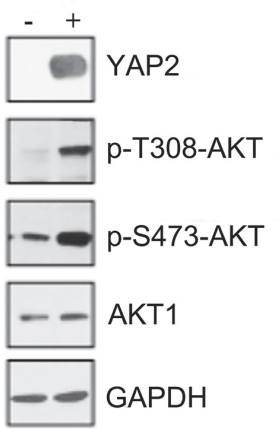

B

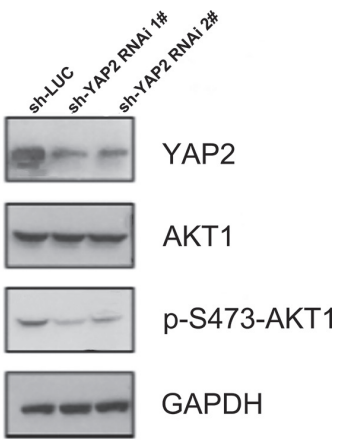

Figure 4. Overexpression of YAP2 upregulate the activity of AKT, and knocking down YAP2 reduces the activity of AKT. A. Expression of YAP2 and AKT in cells treated with cells containing YAP2 adenovirus. B. Relative to wild primitive cardiomyocytes, when using RNAi knockdown of YAP2 resulted in reduced expression of active AKT1. LUC, H9C2 cells labelled with luciferase fluorescein.
Sav, Wts, and Mats. Studies have found that in the Drosophila Hippo signaling pathway regulating organ size and development, MST1 has the same function as Hippo in mammals (Misra and Irvine 2018).

As a member of the MST pathway, Lats can regulate the size of organs (Mo 2017). The leading presence in heart tissue is Lats2. In DN-Lats2 transgenic mice that inhibit endogenous Lats function, an increase in the size of individual cardiomyocytes, hypertrophy of the ventricles, and pathological changes in cardiac function, such as a marked decrease in tolerance to hypertension are observed (Ikeda et al. 2019).

YAP protein is the main target of the MST pathway (Yamauchi and Moroishi 2019). The YAP gene first attracted people's attention in fruit fly experiments. The YAP gene in fruit flies, also known as Yorkie (Yki), encodes a protein that belongs to a transcription factor. Studies have shown that YAP can be involved in cell proliferation and cell cycle regulation at the transcription level. The transcription of the gene promotes cell division and cell survival. Any protein mutation in the Hippo pathway or overexpression of the Yki gene will cause excessive growth of Drosophila eye or wing cells.

As a downstream effector molecule of the MST pathway, the mammalian YAP gene can be phosphorylated by Lats, migrating from the nucleus to the cytoplasm and losing its activity. YAP2 is mainly found in mammalian heart tissue. Therefore, the effect of Lats 2 on cardiomyocytes may be due to the regulation of YAP2 (Xiao et al. 2018). Related studies and our preliminary pre-experiments suggest that YAP2 can act as a transcription factor in the nucleus to regulate cardiomyocyte size. When YAP2 is phosphorylated, it migrates out of the nucleus and becomes an inactive protein, thus losing its regulatory function on decidual myocyte size. 
A

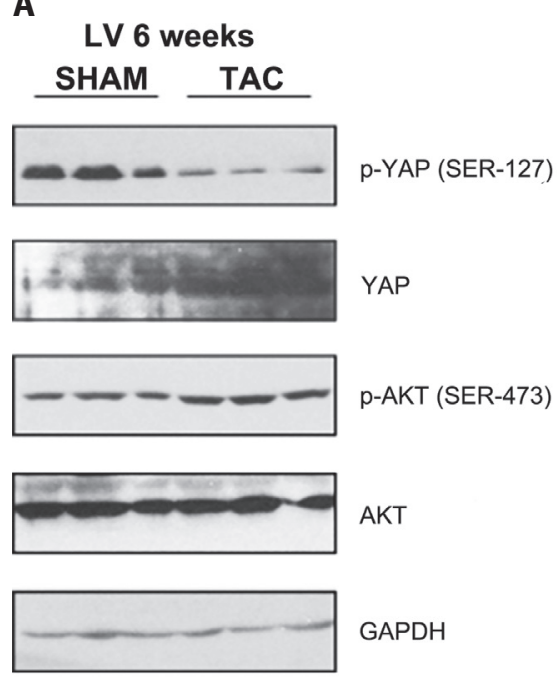

B

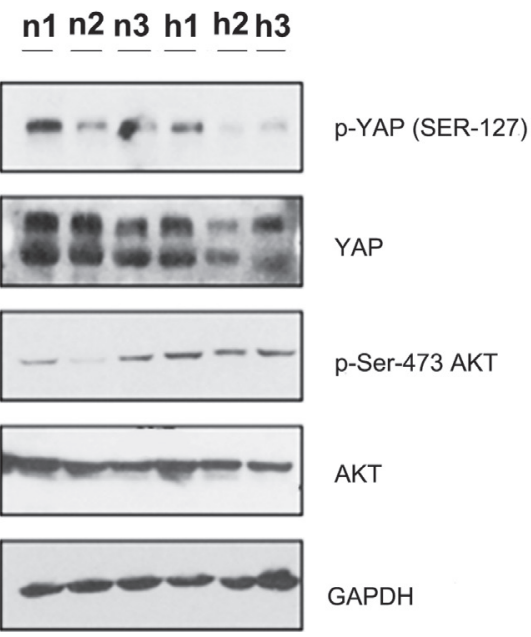

Figure 5. Mouse model of cardiac hypertrophy. A. Mouse HCM model samples show that YAP2 can upregulate AKT1 activity. B. YAP2 is shown to upregulate AKT1 activity expression in samples from patients with HCM. For abbreviations, see Fig. 2.
Thus, our experimental results confirm that the activity of YAP2 is increased in the HCM mouse model.

It was found that there was a change in YAP2 activity in human cardiac muscle tissue (Talman and Ruskoaho 2016). We performed immunohistochemistry to detect the location of YAP2 expression in myocardial tissues of hypertrophic cardiomyopathy and normal humans. The results of intergroup comparisons revealed the presence of variation in YAP2 activity in human myocardial tissues.

We used overexpressed YAP2 virus and GFP control to infect rat cardiac primary cells at the cellular level. As a result, it was shown that overexpression of YAP2 could cause changes in primary cardiomyocyte size and that YAP2 could affect the expression activity of Akt1. Therefore, we suggest that overexpression of YAP2 can cause the enlargement of cardiomyocytes.

In in vitro animal experiments, we compared YAP2 expression, phosphorylation levels of the YAP2-S127 locus, protein levels of YAP2-related genes, and their activity levels in myocardial specimens from a mouse model of TAC and normal control rats. The results revealed that at the animal model level, the protein level and activity of YAP2 were increased in the heart tissue of the mouse model of ascending aortic ligation and that YAP2 could promote cardiomyocyte hypertrophy, upregulating the activity of related genes. Our study of YAP2 and YAP2-S127 phosphorylation levels in human HCM tissue versus normal myocardial specimens showed that YAP2 protein amounts and activity were increased in HCM specimens.

At the animal level, we plan to use the heart of YAP2 cardiomyocyte-specific transgenic rats and knockout rats to analyze the difference of the Microarray gene to obtain the differential gene expression profiles of normal and transgenic or knockout rats. At the same time, we further detect the activity of YAP2 and the level of Akt phosphorylation and protein expression, and screen and identify the proteins and molecular mechanisms that regulate Akt phosphorylation on the regulatory genes of YAP2.

The verification of clinical specimens will also use a similar method to determine the specific molecular mechanism between YAP2 and HCM, provide a theoretical basis for YAP2 as a target for intervention and treatment of this disease. For the study of myocardial hypertrophy, the function and molecular mechanism of genes lay the foundation.

\section{Conclusions}

YAP2 promotes cardiomyocyte hypertrophy in HCM, proving that YAP2 is a new critical factor in the formation of HCM. The final effector YAP2 in the Hippo pathway can affect the activity of downstream related genes by regulating the activity of Akt1, thereby promoting the proliferation and hypertrophy of human cardiomyocytes.

Conflict of interest. The authors declare that they have no competing interests.

\section{References}

Avruch J, Zhou D, Fitamant J, Bardeesy N (2011): Mst1/2 signalling to Yap: gatekeeper for liver size and tumour development. Br. J. Cancer 104, 24-32 https://doi.org/10.1038/sj.bjc.6606011

Azad T, Ghahremani M, Yang X (2019): The role of YAP and TAZ in angiogenesis and vascular mimicry. Cells 8, 407 https://doi.org/10.3390/cells8050407 
Gangadharan B, Sunitha MS, Mukherjee S, Chowdhury RR, Haque F, Sekar N, Sowdhamini R, Spudich JA, Mercer JA (2017): Molecular mechanisms and structural features of cardiomyopathycausing troponin T mutants in the tropomyosin overlap region. Proc. Natl. Acad. Sci. USA 114, 11115-11120 https://doi.org/10.1073/pnas.1710354114

Hall CA, Wang R, Miao J, Oliva E, Shen X, Wheeler T, Hilsenbeck SG, Orsulic S, Goode S (2010): Hippo pathway effector Yap is an ovarian cancer oncogene. Cancer Res. 70, 8517-8525 https://doi.org/10.1158/0008-5472.CAN-10-1242

He J, Gu D, Wu X, Reynolds K, Duan X, Yao C, Wang J, Chen CS, Chen J, Wildma RP (2005): Major causes of death among men and women in China. N. Engl. J. Med. 353, 1124-1134 https://doi.org/10.1056/NEJMsa050467

Ikeda S, Mizushima W, Sciarretta S, Abdellatif M, Zhai P, Mukai R, Fefelova N, Oka SI, Nakamura M, Del Re DP (2019): Hippo deficiency leads to cardiac dysfunction accompanied by cardiomyocyte dedifferentiation during pressure overload. Circ. Res. 124, 292-305 https://doi.org/10.1161/CIRCRESAHA.118.314048

Jiang Q, Liu D, Gong Y, Wang Y, Sun S, Gui Y, Song H (2009): YAP is required for the development of brain, eyes, and neural crest in zebrafish. Biochem. Biophys. Res. Commun. 384, 114-119 https://doi.org/10.1016/j.bbrc.2009.04.070

Liu AM, Xu MZ, Chen J, Poon RT, Luk JM (2010): Targeting YAP and Hippo signaling pathway in liver cancer. Expert Opin. Ther. Targets 14, 855-868 https://doi.org/10.1517/14728222.2010.499361

Matsui Y, Nakano N, Shao D, Gao S, Luo W, Hong C, Zhai P, Holle E, Yu X, Yabuta N (2008): Lats2 is a negative regulator of myocyte size in the heart. Circ. Res. 103, 1309-1318 https://doi.org/10.1161/CIRCRESAHA.108.180042

Misra JR, Irvine KD (2018): The Hippo signaling network and its biological functions. Annu Rev. Genet. 52, 65-87 https://doi.org/10.1146/annurev-genet-120417-031621

Mo JS (2017): The role of extracellular biophysical cues in modulating the Hippo-YAP pathway. BMB Rep. 50, 71-78 https://doi.org/10.5483/BMBRep.2017.50.2.199

Overholtzer M, Zhang J, Smolen GA, Muir B, Li W, Sgroi DC, Deng CX, Brugge JS, Haber DA (2006): Transforming properties of YAP, a candidate oncogene on the chromosome 11q22 amplicon. Proc. Natl. Acad. Sci. USA 103, 12405-12410 https://doi.org/10.1073/pnas.0605579103

Pan D (2007): Hippo signaling in organ size control. Genes Dev. 21, 886-897 https://doi.org/10.1101/gad.1536007

Steinhardt AA, Gayyed MF, Klein AP, Dong J, Maitra A, Pan D, Montgomery EA, Anders RA (2008): Expression of Yesassociated protein in common solid tumors. Hum. Pathol. 39, $1582-1589$ https://doi.org/10.1016/j.humpath.2008.04.012
Talman V, Ruskoaho H (2016): Cardiac fibrosis in myocardial infarction-from repair and remodeling to regeneration. Cell Tissue Res. 365, 563-581 https://doi.org/10.1007/s00441-016-2431-9

Wang Y, Dong Q, Zhang Q, Li Z, Wang E, Qiu X (2010): Overexpression of yes-associated protein contributes to progression and poor prognosis of non-small-cell lung cancer. Cancer Sci. 101, 1279-1285 https://doi.org/10.1111/j.1349-7006.2010.01511.x

Wong CX, Brown A, Lau DH, Chugh SS, Albert CM, Kalman JM, Sanders P (2019): Epidemiology of sudden cardiac death: global and regional perspectives. Heart Lung Circ. 28, 6-14 https://doi.org/10.1016/j.hlc.2018.08.026

Xiao Y, Hill MC, Zhang M, Martin TJ, Morikawa Y, Wang S, Moise AR, Wythe JD, Martin JF (2018): Hippo signaling plays an essential role in cell state transitions during cardiac fibroblast development. Dev. Cell. 45, 153-169 https://doi.org/10.1016/j.devcel.2018.03.019

Yamauchi T, Moroishi T (2019): Hippo pathway in mammalian adaptive immune system. Cells 8, 398 https://doi.org/10.3390/cells8050398

Yang W, Han W, Qin A, Wang Z, Xu J, Qian Y (2018): The emerging role of Hippo signaling pathway in regulating osteoclast formation. J. Cell. Physiol. 233, 4606-4617 https://doi.org/10.1002/jcp.26372

Zhang J, Smolen GA, Haber DA (2008): Negative regulation of YAP by LATS1 underscores evolutionary conservation of the Drosophila Hippo pathway. Cancer Res. 68, 2789-2794 https://doi.org/10.1158/0008-5472.CAN-07-6205

Zhang X, George J, Deb S, Degoutin JL, Takano EA, Fox SB, AOCS Study group, Bowtell DD, Harvey KF (2011): The Hippo pathway transcriptional co-activator, YAP, is an ovarian cancer oncogene. Oncogene 30, 2810-2822 https://doi.org/10.1038/onc.2011.8

Zhao B, Lei QY, Guan KL (2008): The Hippo-YAP pathway: new connections between regulation of organ size and cancer. Curr. Opin. Cell Biol. 20, 638-646 https://doi.org/10.1016/j.ceb.2008.10.001

Zhao B, Li L, Lei Q, Guan KL (2010): The Hippo-YAP pathway in organ size control and tumorigenesis: an updated version. Genes Dev. 24, 862-874 https://doi.org/10.1101/gad.1909210

Zheng Y, Wang W, Liu B, Deng H, Uster E, Pan D (2015): Identification of happyhour/MAP4K as alternative Hpo/Mst-like kinases in the hippo kinase cascade. Dev Cell. 34, 642-655 https://doi.org/10.1016/j.devcel.2015.08.014

Zhou J (2014): An emerging role for Hippo-YAP signaling in cardiovascular development. J. Biomed. Res. 28, 251-254

Received: June 8, 2021

Final version accepted: July 14, 2021 